Editorial: Hospital Metropolitano

ISSN (impreso) 1390-2989 - ISSN (electrónico) 2737-6303

Edición: Vol. 29 No 1 (2021) enero-marzo

DOI: https://doi.org/10.47464/MetroCiencia/vol29/1/2021/16-22

URL: https://revistametrociencia.com.ec/index.php/revista/article/view/132

Pág: $16-22$

\title{
Prevalencia de bloqueo residual en la Unidad de Cuidados Postanestésicos del Hospital Metropolitano
}

\section{Prevalence of residual blockade in the Postanesthetic Care Unit of the Hospital Metropolitano}

\author{
Xavier Raúl Mantilla Pinto' ${ }^{1 D}$; Carla Belén Dávila Estévez ${ }^{2}$; \\ Adriana Gabriela Terán Calderón ${ }^{2}$ \\ Servicio de Anestesiolog ía, Hospital Metropolitano, Quito, Ecuador ${ }^{1}$ \\ Posgrado de Anestesiolog ía, Reanimación y Terapia del Dolor, Pontificia Universidad Católica del Ecuador, Quito, Ecuador ${ }^{2}$
}

Recibido: 01/12/2020 Aceptado: 20/12/2020 Publicado: 29/01/2021

\section{RESUMEN}

El bloqueo residual se define como la debilidad muscular postoperatoria producida por un antagonismo incompleto de los relajantes neuromusculares, siendo sus principales complicaciones las respiratorias. Actualmente, el mejor método de evaluación objetiva del bloqueo neuromuscular es el Tren de Cuatro (TOF). El objetivo de este estudio fue describir la prevalencia de bloqueo residual en la Unidad de Cuidados Postanestésicos (UCPA) en pacientes ASA I y ASA II sometidos a cirugía electiva y de emergencia bajo anestesia general en el Hospital Metropolitano. Se realizó un estudio observacional, descriptivo y prospectivo en 211 sujetos, donde mediante el TOF se determinó que la prevalencia de bloqueo residual en la UCPA fue del 8,5\%, valor que se relacionó con la falta de monitorización de la función neuromuscular, determinando además que el empleo de fármacos que revierten el bloqueo neuromuscular no excluye la presencia de bloqueo residual. Se recomienda el uso rutinario de monitorización de la relajación neuromuscular para prevenir y diagnosticar el bloqueo residual, además de la administración de reversión farmacológica independientemente del tiempo quirúrgico.

Palabras claves: Bloqueantes neuromusculares, monitorización del bloqueo neuromuscular, bloqueo residual, tren de cuatro.

\section{ABSTRACT}

Residual blockade is defined as postoperative muscle weakness caused by incomplete antagonism of neuromuscular relaxants, its main complications being respiratory, currently the best method of objective evaluation of neuromuscular blockade is the Train of Four (TOF). The objective of this study was to describe the prevalence of residual block in the Post Anesthesia Care Unit (UCPA) in patients with ASA and ASA II undergoing elective and emergency surgery under general anesthesia at the Metropolitan Hospital. An observational, descriptive and prospective study was conducted in 211 subjects, where it was determined that the prevalence of residual block in UCPA was $8.5 \%$ with TOF, a value that was related to the lack of monitoring of neuromuscular function, also determining that the use of drugs that reverse neuromuscular blockade does not exclude the presence of residual blockade. Routine use of neuromuscular relaxation monitoring is recommended to prevent and diagnose residual block, in addition to drug reversal administration regardless of surgical time.

Keywords: Neuromuscular blockers, monitoring of neuromuscular block, residual blockade, train of four.

IDs Orcid

https://orcid.org/0000-0003-4074-6811 https://orcid.org/0000-0001-8613-8040 https://orcid.org/0000-0001-7519-4194

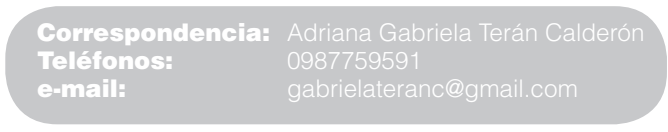




\section{INTRODUCCIÓN}

La anestesia general es un proceso reversible siendo una acción médica controlada en la que se produce la ausencia temporal de la sensibilidad táctil y dolorosa a través de fármacos, se caracteriza por cuatro estados fisiológicos y conductuales: inconsciencia, amnesia, analgesia y abolición de reflejos ${ }^{1}$.

Los relajantes neuromusculares son fármacos que se utilizan en una anestesia general para facilitar la intubación orotraqueal, permitir una adecuada ventilación mecánica y proporcionar mejores condiciones para el procedimiento quirúrgico². Los fármacos más empleados son los de acción intermedia, como el rocuronio ${ }^{3}$. El despertar y la extubación del paciente (emergencia de la anestesia general) al finalizar la cirugía debe realizarse una vez que se haya recuperado del bloqueo neuromuscular, para su posterior traslado a la Unidad de Cuidados Postanestésicos $(\text { UCPA })^{4}$.

El control de la función neuromuscular debe ser medido de una manera objetiva, mediante la evaluación eléctrica de la respuesta neuromuscular a la estimulación de un nervio periférico motor ${ }^{5}$. El Tren de Cuatro o TOF, por sus siglas en inglés, se introdujo en la década de los 70, constituyendo el mejor método de evaluación objetiva del bloqueo neuromuscular5,6; se realiza mediante cuatro estímulos administrados cada 0,5 segundos con una frecuencia de $2 \mathrm{~Hz}$, se repite cada 10 o 20 segundos, y se calcula dividiendo la amplitud del T4 o cuarta respuesta por la amplitud del T1 o primera respuesta ${ }^{7}$, constituyendo la proporción TOF de $1^{8}$.

Un valor de TOF > 0.9 determina la ausencia de un bloqueo neuromuscular residual clínicamente notable ${ }^{6}$. Una estimación de TOF adecuada asegura los reflejos protectores de la vía aérea ${ }^{9}$. Es el método que en comparación con la evaluación clínica subjetiva permite un correcto diagnóstico del bloqueo residual ${ }^{5}$.

El bloqueo neuromuscular residual, se define como la persistencia de la parálisis o debilidad muscular una vez terminada la cirugía, producto de un antagonismo incompleto de los bloqueantes neuromusculares, es una condición clínica que se caracteriza por impedir la transmisión neuromuscular, lo que se traduce en una alteración funcional motora ${ }^{5}$.

Son varias las causas que pueden provocar la persistencia del bloqueo residual neuromuscular, las mismas que deben ser analizadas desde el preoperatorio de forma individual en cada paciente que ingresa a sala de operaciones ${ }^{9}$. Dentro de estos factores se puede mencionar: la edad del paciente, sexo, Índice de Masa Corporal (IMC), comorbilidades, estratificación ASA, tiempo quirúrgico, tipo de cirugía, hipoter- mia, dosis total de bloqueante neuromuscular (BNM), uso de drogas reversoras del bloqueo neuromuscu$\operatorname{lar}^{10}$.

Los anticolinesterásicos como la neostigmina y las ciclodextrinas como el sugammadex, son los fármacos manejados para la reversión del bloqueo neuromuscular ${ }^{11,12}$. El empleo de los fármacos reversores constituye una de las varias estrategias para evitar el efecto residual de los relajantes neuromusculares ${ }^{13}$.

En pacientes sometidos a un procedimiento quirúrgico en los que se utilizó bloqueantes neuromusculares no despolarizantes, existe una incidencia de bloqueo residual correspondiente al 88\% durante la extubación endotraqueal, mientras que a la llegada a la UCPA es del 83\% ${ }^{14,15}$; sin embargo, la literatura describe una amplia variabilidad de presentación de relajación residual en la UCPA que oscila entre el 5 y el $93 \% 16$.

Aun cuando existen varios estudios sobre la incidencia de relajación residual, ésta continúa estando presente en la UCPA. Existen diferentes publicaciones científicas donde se registra una alta incidencia de relajación residual, con un porcentaje de entre el 5\% y el $93 \%$ presente en los pacientes que ingresan a la UCPA², desarrollando complicaciones postoperatorias importantes que aumentan la morbimortalidad de los pacientes ${ }^{10}$. La principal complicación que se ha descrito es la respiratoria, mencionando a la neumonía como la patología más común que podría presentarse $^{17}$.

Al establecer que la existencia de bloqueo residual en la UCPA conlleva un incremento en la morbimortalidad de los pacientes, el objetivo principal del presente estudio es determinar la prevalencia de bloqueo residual en la UCPA en pacientes ASA I y ASA Il sometidos a cirugía electiva y de emergencia bajo anestesia general en el Hospital Metropolitano, con el fin de evaluar una estadística actual, analizar los resultados obtenidos y formular recomendaciones que nos ayuden a prevenir el bloqueo residual.

\section{MATERIALES Y MÉTODOS}

Se realizó un estudio observacional, de tipo descriptivo, prospectivo en 211 sujetos en el período comprendido desde el 01 de abril al 31 de mayo del 2020, donde se incluyó a pacientes de sexo femenino y masculino, estado físico ASA I y ASA II, con edad igual o mayor a 18 años, sometidos a cirugía electiva y de emergencia bajo anestesia general con administración previa de relajantes neuromusculares no despolarizantes y con una estancia mínima de una hora en la UCPA. Se excluyeron a los pacientes con estado físico ASA III, ASA IV, ASA V y ASA VI, bajo anestesia regional o local, con enfermedades neuromusculares, lesiones de nervio cubital y con amputación de miembros superiores. 
El anestesiólogo encargado de cada paciente fue responsable de la elección de la técnica anestésica, fármacos administrados y la decisión de colocar monitorización de la función neuromuscular durante el trans-anestésico.

Inmediatamente a la llegada de los pacientes seleccionados en la UCPA, previa colocación de oxígeno y monitorización de signos vitales, se realizó la medición de la estimulación nerviosa mediante el equipo TOF WATCH S. Se fijó el miembro superior a estimularse en posición de supinación, permitiendo la movilidad del dedo pulgar e inmovilizando los restantes cuatro dedos, se colocaron sobre el nervio cubital dos electrodos en el lado volar de la muñeca.

El electrodo distal se colocó aproximadamente a $1 \mathrm{~cm}$ proximal al punto en el que el pliegue flexor proximal de la muñeca atraviesa el lado radial del tendón del músculo flexor cubital del carpo, y el electrodo proximal se colocó de modo que la distancia entre los centros de ambos electrodos sea entre 3 y $6 \mathrm{~cm}$. La estimulación eléctrica que se consiguió fue la flexión digital y la aducción del dedo pulgar del miembro superior.

Las variables recolectadas como edad, sexo, valor TOF obtenido en la UCPA, monitorización de la función neuromuscular durante el transanestésico, reversión del bloqueo neuromuscular, duración total de anestesia y comorbilidades presentes asociadas a bloqueo residual, se concentraron en una base de datos en Microsoft Excel y posteriormente fueron tabulados en el programa estadístico SPSS, se estimó las frecuencias absolutas, relativas y porcentajes.

\section{RESULTADOS}

En este estudio participaron 211 pacientes ASA I y ASA II sometidos a cirugía electiva o de emergencia bajo anestesia general en los meses de abril y mayo en la unidad de cuidados postanestésicos del Hospital Metropolitano, en el año 2020. Se determinó que el 91,5\% presentó un valor de TOF mayor o igual a 90 , mientras el $8,5 \%$ obtuvo un valor de TOF menor o igual a 89, es decir, presentaron bloqueo neuromuscular residual. (Figura 1).

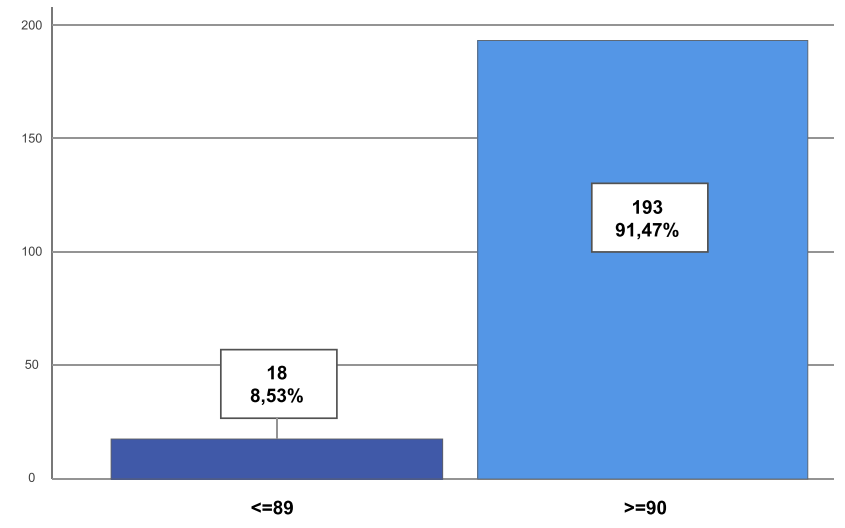

Figura 1. Valor TOF obtenido en la UCPA.
En la tabla 1 se muestran los valores de TOF obtenidos en los 18 pacientes (8,53\%) que presentaron relajación neuromuscular residual.

Tabla 1. Valores TOF en pacientes con bloqueo neuromuscular residual.

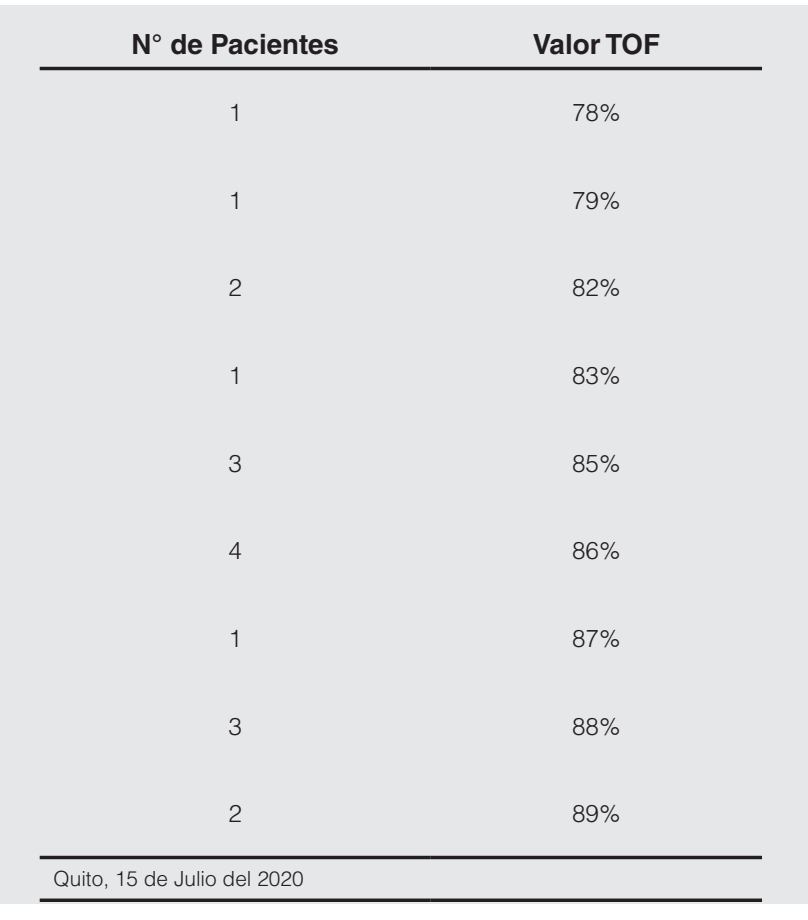

Fuente: Base de datos del proyecto de investigación.

En el $75,3 \%(n=159)$ de los procedimientos quirúrgicos incluidos en nuestro estudio no se utilizó monitorización neuromuscular durante el intraoperatorio, pero en el $24,6 \%(n=72)$ de las cirugías sí fue utilizada.

En cuanto a la distribución por sexo de los participantes fue de 128 mujeres $(60,7 \%)$ y 83 hombres $(39,3 \%)$, la edad fue asociada en tres grupos etarios, el primer grupo corresponde a pacientes entre 18 a 39 años con 100 casos $(47,4 \%)$, el segundo grupo corresponde a pacientes entre 40 a 64 años con 83 casos (39.3\%), y el tercer grupo correspondiente a pacientes entre 65 a 81 años con 28 casos (13,3\%).

Así, se analizó el grupo etario en relación a la presencia de bloqueo residual (Tabla 2) determinando que el grupo de entre 65 y 81 años presentó mayor frecuencia de bloqueo residual con un porcentaje de $3,3 \%(n=7)$, seguido por el grupo etario de entre 40 y 64 años con $2,8 \%(n=6)$ y con un $2,4 \%(n=5)$ en el grupo de 18 a 39 años, con una relación estadísticamente significativa $(p=\leq 0,003)$. 
Tabla 2. Bloqueo neuromuscular residual y edad.

\begin{tabular}{|c|c|c|c|c|c|}
\hline & & & \multicolumn{2}{|c|}{ Valor TOF en la UCPA } & \multirow{2}{*}{ Total } \\
\hline & & & $<=89$ & $>=90$ & \\
\hline \multirow{6}{*}{$\begin{array}{c}\text { Edad en } \\
\text { grupos (años) }\end{array}$} & \multirow{2}{*}{$18-39$} & Recuento & 5 & 95 & 100 \\
\hline & & $\%$ del total & $2,4 \%$ & $45,0 \%$ & $47,4 \%$ \\
\hline & \multirow{2}{*}{$40-64$} & Recuento & 6 & 77 & 83 \\
\hline & & $\%$ del total & $2,8 \%$ & $36,5 \%$ & $39,3 \%$ \\
\hline & \multirow{2}{*}{$65-81$} & Recuento & 7 & 21 & 28 \\
\hline & & $\%$ del total & $3,3 \%$ & $10,0 \%$ & $13,3 \%$ \\
\hline \multirow{2}{*}{\multicolumn{2}{|c|}{ Total }} & Recuento & 18 & 193 & 211 \\
\hline & & $\%$ del total & $8,5 \%$ & $91,5 \%$ & $100,0 \%$ \\
\hline \multicolumn{6}{|c|}{ chi $^{2}: 11,511 \quad$ p: $\leq 0.003$} \\
\hline \multicolumn{6}{|c|}{ Quito, 15 de julio del 2020} \\
\hline
\end{tabular}

Fuente: Base de datos del proyecto de investigación.

Un hallazgo fundamental fue que en el $75,3 \%$ de los procedimientos quirúrgicos realizados y estudiados, no se utilizó monitorización neuromuscular durante el intraoperatorio y únicamente en el $24,6 \%$ de las cirugías sí fue utilizada. (Figura 2).

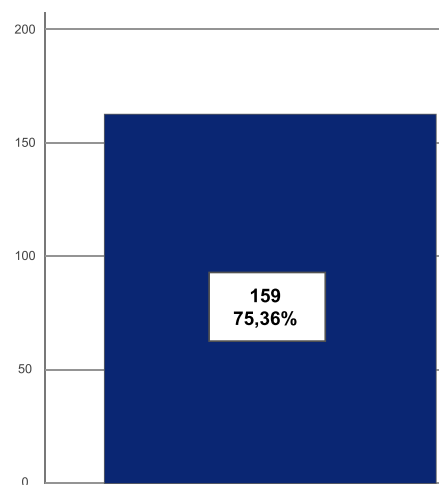

No

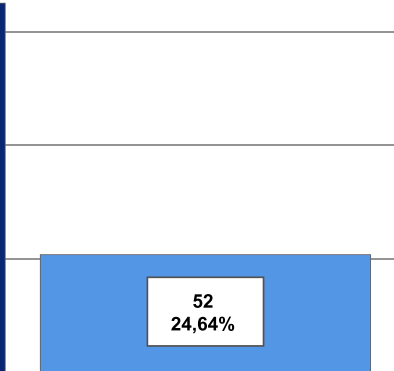

SI
Figura 2. Monitorización neuromuscular intraoperatoria

En un $81 \%$ (171 casos) se utilizó el fármaco sugammadex para la reversión del bloqueo neuromuscular, mientras que en un 4,7\% (10 casos) se utilizó neostigmina y el 14,2\% (30 casos) no recibieron ningún fármaco de reversión neuromuscular. (Figura 3). Además, se evidenció un 2,4\% de pacientes a quienes se le administró sugammadex y que al no estar monitorizados presentaron relajación residual en la UCPA.

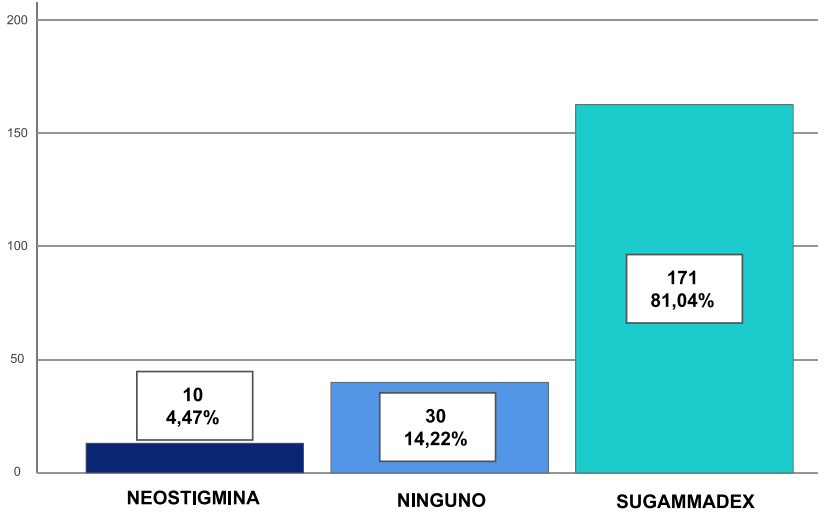

Figura 3. Reversión de bloqueo neuromuscular.

Predominantemente, la mayor parte de las cirugías se efectuaron en un tiempo que osciló entre 61 y 120 minutos (46,4\%), la duración total de la anestesia de los procedimientos quirúrgicos analizados se puede observar a continuación en la tabla 3.

Tabla 3. Duración total de anestesia (minutos).

\begin{tabular}{cc}
$\begin{array}{c}\text { Duración Total de } \\
\text { Anestesia (minutos) }\end{array}$ & $\begin{array}{c}\text { Porcentaje de } \\
\text { Pacientes }\end{array}$ \\
\hline$<60$ minutos & $34,6 \%$ \\
$61-120$ minutos & $46,45 \%$ \\
$121-180$ minutos & $12,80 \%$ \\
$181-240$ minutos & $2,37 \%$ \\
$241-300$ minutos & $2,37 \%$ \\
$>301$ minutos & $1,42 \%$ \\
\hline Quito, 15 de julio del 2020 & \\
\hline
\end{tabular}

Fuente: Base de datos del proyecto de investigación. 
Además, la Diabetes mellitus 2 estuvo presente en el 6,1\% de los pacientes analizados, seguida de la Obesidad con el 3,7\%, la Falla Hepática y la Falla Renal se presentó con similitud en el 1,4\% respectivamente, la Enfermedad Pulmonar Obstructiva Crónica en el 0,9\%, sin embargo, el 86,2\% de los pacientes, no presentó ninguna comorbilidad que se haya asociado con el Bloqueo Neuromuscular Residual. (Figura 4).

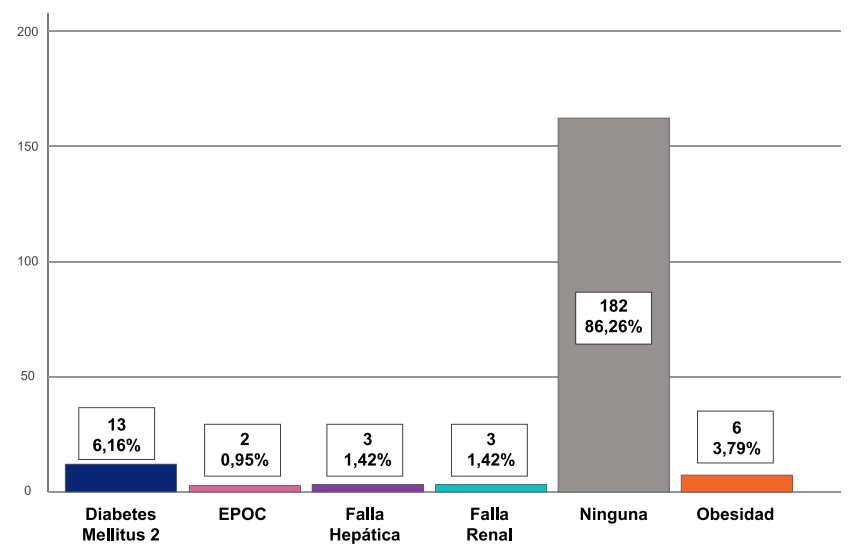

Figura 4. Presencia de comorbilidades asociadas a bloqueo neuromuscular residual.

\section{DISCuSIón}

Los bloqueantes neuromusculares son de uso frecuente en los centros quirúrgicos, tomando en cuenta que la anestesia general es la técnica anestésica elegida en las dos terceras partes de las cirugías a nivel mundial y que en más de la mitad de ellas se usa bloqueantes neuromusculares ${ }^{9}$. Su implementación, si bien tiene grandes beneficios en la anestesia, como el proceder adecuado de la laringoscopia, el acoplamiento del paciente a la ventilación mecánica o un plano quirúrgico adecuado, la falta de monitorización del efecto de estos fármacos puede provocar un aumento significativo en la incidencia de relajación residual².

Existen diferentes publicaciones científicas donde se registra una alta incidencia de relajación residual, con un porcentaje de entre el $5 \%$ y $93 \%$ presente en los pacientes que ingresan a la UCPA ${ }^{16}$, desarrollando complicaciones postoperatorias importantes, por lo que se destaca como punto primordial la utilización adecuada y regular de la monitorización de bloqueo neuromuscular durante la anestesia y una vez que ésta ha concluido, además de la administración de reversión del bloqueo neuromuscular ${ }^{18}$.

Los datos de este estudio arrojan una prevalencia del $8,5 \%$ de bloqueo residual en la UCPA, valor comparable al reportado en un artículo anterior elaborado por Cifuentes en el Hospital Metropolitano, donde se reportó una incidencia del $11 \%{ }^{19}$. El método de monitorización neuromuscular en la UCPA elegido para la realización de nuestro estudio fue el TOF debido a su facilidad de aplicación e interpretación de resultados, se cumplió con los lineamientos para su correcta utilización teniendo en cuenta los voltajes requeridos para la correcta valoración de la contracción muscular en pacientes despiertos.

A pesar de existir la recomendación establecida de realizar una regular monitorización transquirúrgica del estado de relajación residual, como lo menciona Viby-Mogensen, donde se determina que la monitorización neuromuscular incrementa la seguridad del paciente y previene la incidencia de bloqueo residual $^{5}$, en nuestro proyecto de investigación se determinó que sólo el 22,7\% de los pacientes incluidos en este estudio cumplieron con el estándar de monitorización necesaria en una anestesia general con el uso de relajantes neuromusculares.

La administración de medicamentos que reviertan el estado de relajación residual fue determinada por cada anestesiólogo, observando que la mayoría de ellos, es decir el 81,04\% optó por el sugammadex como fármaco específico para la reversión de la acción del rocuronio; sin embargo, en varios estudios científicos se menciona que el uso de fármacos que reviertan el efecto de los relajantes neuromusculares no asegura una prevención absoluta de bloqueo residual. Es así que en la investigación de Mari-Zapata realizada en la ciudad de México en el año 2016 se menciona una prevalencia del $2,48 \%$ de bloqueo residual a pesar de la administración de sugammadex ${ }^{6}$.

En el análisis demográfico de nuestro estudio se observa que existe una prevalencia mayor de bloqueo residual en los pacientes con edades entre 65 y 81 años, lo que es comparable a los hallazgos bibliográficos obtenidos en la presente investigación. De hecho, en el estudio publicado por la Revista Colombiana de Anestesiología en el año 2016, se menciona una incidencia superior de relajación residual en pacientes entre 65 y 89 años, en comparación con pacientes entre 19 y 57 años (44 vs. $20 \%)^{20}$.

Exponemos además, los tipos de comorbilidades más frecuentes relacionadas a bloqueo residual que presentaron los pacientes del estudio. Como referencia revisamos el artículo de la Sociedad Española de Anestesiología y Reanimación publicado en el 2019 por Brull, S.J., donde se menciona la prevalencia de bloqueo residual en pacientes vulnerables, destacando a la obesidad como factor de riesgo de presentar complicaciones pulmonares postoperatorias por bloqueo residual ${ }^{21}$; sin embargo, en nuestra investigación la Diabetes mellitus 2 obtuvo una prevalencia del 1,4\%, siendo la comorbilidad más frecuente en los pacientes con bloqueo residual. 
Este estudio representa la evaluación de la correcta monitorización de la relajación neuromuscular durante la anestesia general y el uso adecuado de fármacos que reviertan este estado, en el escenario de un hospital privado-docente, en donde se cuenta con monitorización adecuada en cada sala de operaciones y además se tiene al alcance las drogas necesarias para revertir la relajación neuromuscular.

\section{CONCLUSIONES}

El presente estudio reveló que la prevalencia de bloqueo residual en la UCPA del Hospital Metropolitano de Quito fue del 8,5\%, un índice bajo en relación con los estudios descritos mundialmente.

La falta de monitorización objetiva de la relajación neuromuscular intraoperatoria aumenta significativamente el riesgo de presentar bloqueo residual, y la utilización de fármacos para la reversión del bloqueo neuromuscular por sí solo, no excluye la presencia de bloqueo residual, si no se monitoriza objetivamente al paciente.

También, el fármaco más utilizado para la reversión del bloqueo neuromuscular fue sugammadex, el cual al poseer una afinidad selectiva por el rocuronio y anular su efecto, contribuye a que la prevalencia de relajación residual sea baja; sin embargo, en nuestro estudio se evidenció un 2,9\% de pacientes a quienes se les administró sugammadex y que al no estar monitorizados presentaron relajación residual en la UCPA.

La utilización de fármacos para la reversión del bloqueo neuromuscular se debe realizar independientemente del tiempo quirúrgico que haya transcurrido posterior a la dosis de administración del relajante neuromuscular.

Los pacientes adultos mayores presentaron más riesgo de relajación residual, así como los pacientes ASAll, además, se demostró que la comorbilidad más frecuente asociada a bloqueo neuromuscular residual fue la Diabetes mellitus 2, sin embargo, pacientes sin patologías asociadas también desarrollaron bloqueo residual en la UCPA.

\section{CONTRIBUCIÓN DE CADA AUTOR}

XM revisión general del artículo.

CD concepto, redacción del manuscrito, análisis e interpretación de los datos.

GT diseño, redacción del manuscrito, análisis e interpretación de los datos.

\section{FINANCIACIÓN PROPIA O NINGUNA}

Declaramos que la financiación del trabajo de investigación fue propia.

\section{CONFLICTOS DE INTERÉS}

Los autores declaran no tener conflictos de interés de ningún tipo.

\section{AGRADECIMIENTOS}

Agradecemos al Hospital Metropolitano, de manera particular al Servicio de Anestesiología y a su personal por facilitar y apoyar el desarrollo el estudio.

\section{REFERENCIAS BIBLIOGRÁFICAS}

1. Brown E. Control del estado encefálico durante la anestesia general y la sedación. En: Miller R, Cohen N, Ericksson L, Fleisher L, Wiener - Kronish K, Young Y. Miller Anestesia. Octava Edición. Barcelona: Elsevier; 2016. 1524-1540

2. Murphy G, Szokol J, Avram M, Greenberg S, Shear T, Vender J et al. Residual Neuromuscular Block in the Elderly. Anesthesiology. 2015;123(6):1322-1336

3. Naguib M, Lien C, Meistelman C. Farmacología de los Bloqueantes Neuromusculares. En: Miller R, Cohen N, Erikson L, Fleisher L, Wiener J, Young W. Miller Anestesia. Octava Edición. Barcelona: Elsevier; 2016. 958-994.

4. Hunter J. Reversal of residual neuromuscular block: complications associated with perioperative management of muscle relaxation. British Journal of Anaesthesia. 2017;119: i53-i62.

5. Viby-Mogensen J, Claudius C. Monitorización Neuromuscular. En MiIler R, Cohen N, Eriksson L, Fleisher L, Wiener J, Young W. Miller Anestesia. Octava Edición. Barcelona: Elsevier; 2016. 1604-1621.

6. Mari - Zapata D, De la Torre R, Aguirre C, Álvarez G, Ocampo A, Gutierrez $\mathrm{C}$. Bloqueo residual neuromuscular en pacientes hospalizados versus ambulatorios en la Unidad de Cuidados Postanestésicos. Revista Mexicana de Anestesiología. 2016 Abril - Junio; 39(2): 97-105.

7. Naguib M, Brull S, Kopman A, Hunter J, Fülesdi B, Arkes H et al. Consensus Statement on Perioperative Use of Neuromuscular Monitoring. Anesthesia \& Analgesia. 2018;127(1):71-80.

8. Thilen S, Bhananker S. Qualitative Neuromuscular Monitoring: How to Optimize the Use of a Peripheral Nerve Stimulator to Reduce the Risk of Residual Neuromuscular Blockade. Current Anesthesiology Reports. 2016; 6(2):164-169

9. Ariza F, Dorado F, Enríquez L, González V, Gómez J, Chaparro-Mendoza K et al. Relajación residual postoperatoria en la unidad de cuidados postanestésicos de un hospital universitario: estudio de corte transversal. Revista Colombiana de Anestesiología. 2017; 45(1):1521.

10. Fortier L, McKeen D, Turner K, de Médicis É, Warriner B, Jones P et al. The RECITE Study. Survey of Anesthesiology. 2016;60(2):75.

11. Tajaate N, Schreiber J, Fuchs-Buder T, Jelting Y, Kranke P. Neostigmine-based reversal of intermediate acting neuromuscular blocking agents to prevent postoperative residual paralysis. European Journal of Anaesthesiology. 2018;35(3):184-192.

12. Brueckmann B, Sasaki N, Grobara P, Li M, Woo T, de Bie J et al. Effects of Sugammadex on Incidence of Postoperative Residual Neuromuscular Blockade. Survey of Anesthesiology. 2015;60(4):167-168.

13. Murphy G, Kopman A. Neostigmine as an antagonist of residual block: best practices do not guarantee predictable results. British Journal of Anaesthesia. 2018;121(2):335-337

14. Buwei $Y$, Baoyi O, Shenhjin G, Yan L, Jun L, Dongmel $N$ et al. Incidence of postoperative residual neuromuscular blockade after general anesthesia: A prospective, multicenter, anesthetists - blind, observational study. Current Medical Research and Opinion. 2015:1- 25.

15. Plaud B, Baillard C, Bourgain J, Bouroche G, Desplanque L, Devys $J$ et al. Guidelines on muscle relaxants and reversal in anaesthesia. Anaesthesia Critical Care \& Pain Medicine. 2020;39(1):125-142.

16. Murphy G, De Boer H, Ericksson L, Miller R. Reversión (antagonismo) del bloqueo neuromuscular. En: Miller R, Cohen N, Ericksson L, Fleisher L, Wiener - Kronish J, Young W. Miller Anestesia. Octava Edición. Barcelona: Elsevier; 2016. 994-1027. 
17. Bulka C, Terekhov M, Martin V, Dmochowsky R, Hayes R, Ehrenfelt J. Nondepolarizing Neuromuscular Blocking Agents, Reversal, and Risk of Postoperative Pneumonia. Anesthesiology. 2016 January; 125(4): 647-655.

18. Rudolph M, Chitilian $\mathrm{H}, \mathrm{Ng}$ P, Timm F, Agarwala A, Doney A et al. Implementation of a new strategy to improve the peri-operative management of neuromuscular blockade and its effects on postoperative pulmonary complications. Anaesthesia. 2018;73(9):1067-1078.

19. Cifuentes $G$, Altamirano F. Incidencia de la relajación neuromuscular residual postoperatoria valorada por aceleromiografía en el Hospital Metropolitano. Revista Metro Ciencia. 2011;20(2):70-74
20. González-Cárdenas V, Salazar-Ramírez K, Coral-Sánchez G. Relajación residual postoperatoria en pacientes mayores de 65 años en la Unidad de Cuidado Postanestésico. Revista Colombiana de Anestesiología. 2016;44(3):211-217

21. Brull S, Kopman A. Current Status of Neuromuscular Reversal and Monitoring. Anesthesiology. 2017;126(1):173-190. 